\title{
In-situ acceleration of subrelativistic electrons in the Coma halo and the halo's influence on the Sunyaev-Zeldovich effect ${ }^{\star}$
}

\author{
V. A. Dogiel ${ }^{1,2}$, S. Colafrancesco ${ }^{3}$, C. M. Ko ${ }^{1}$, P. H. Kuo ${ }^{1}$, C. Y. Hwang ${ }^{1}$, W. H. Ip ${ }^{1}$, \\ M. Birkinshaw ${ }^{4}$, and D. A. Prokhorov 5 \\ ${ }^{1}$ Institute of Astronomy, National Central University, Chung-Li 32054, Taiwan \\ 2 I. E. Tamm Theoretical Physics Division, P. N. Lebedev Physical Institute, 117924 Moscow, Russia \\ e-mail: dogiel@lpi.ru \\ 3 INAF - Osservatorio Astronomico di Roma, via Frascati 33, 00040 Monteporzio, Italy \\ 4 Department of Physics, University of Bristol, Tyndall Avenue, Bristol BS8 1TL, UK \\ 5 Moscow Institute of Physics and Technology, Institutskii lane, 141700 Moscow Region, Dolgoprudnii, Russia
}

Received 23 August 2005 / Accepted 19 September 2006

\section{ABSTRACT}

\begin{abstract}
Aims. The stochastic acceleration of subrelativistic electrons from a background plasma is studied in order to find a possible explanation of the hard X-ray emission detected from the Coma cluster.

Methods. We calculate the necessary energy supply as a function of the plasma temperature and of the electron energy, and we show that, for the same value of the hard X-ray flux, the energy supply changes gradually from its high value for the case when emitting particle are non-thermal to lower values when the electrons are thermal. The kinetic equations we use include terms describing particle thermalization as well as momentum diffusion due to the Fermi II acceleration.

Results. We show that the temporal evolution of the particle distribution function has, at its final stationary stage, a rather specific form. This distribution function cannot be described by simple exponential or power-law expressions. A broad transfer region is formed by Coulomb collisions at energies between the Maxwellian and power-law parts of the distribution functions. In this region the radiative lifetime of a single quasi-thermal electron differs greatly from the lifetime of the distribution function as a whole. For a plasma temperature of $8 \mathrm{keV}$, the particles emitting bremsstrahlung at $20-80 \mathrm{keV}$ lie in this quasi-thermal regime. We show that the energy supply required by quasi-thermal electrons to produce the observed hard X-ray flux from Coma is one or two orders of magnitude smaller than the value derived from the assumption of a nonthermal origin of the emitting particles. This result may solve the problem of rapid cluster overheating by nonthermal electrons raised by Petrosian (2001): while Petrosian's estimates are correct for nonthermal particles, they are inapplicable in the quasi-thermal range. We finally analyze the change in Coma's Sunyaev-Zeldovich effect caused by the implied distortions of the Maxwellian spectrum of electrons, and we show that evidence for the acceleration of subrelativistic electrons can, in principle, be derived from detailed spectral measurements.
\end{abstract}

Key words. galaxies: clusters: general - acceleration of particles

\section{Introduction}

An excess of hard X-ray (hereafter HXR) emission above the thermal spectrum has been found in the energy range $20-80 \mathrm{keV}$ from the Coma cluster of galaxies (Fusco-Femiano et al. 1999; Rephaeli \& Gruber 2002; Fusco-Femiano et al. 2004). The validity of this excess is, however, still unclear because Rossetti \& Molendi (2004) re-analyzed the Coma data and found no evidence for such hard X-ray excess. Further re-analysis of the same data (Fusco-Femiano et al. 2004) seem to confirm the presence of the HXR excess at the level observed by Fusco-Femiano et al. (1999) and Rephaeli \& Gruber (2002). The recent INTEGRAL observation of Coma (Renaud et al. 2006) analyzed the morphology of the cluster in the range $18-30 \mathrm{keV}$ and found that the hard X-ray emission comes from an extended source with a radius $\sim 30$ arcmin. The spatial distribution is similar to the thermal one as obtained with XMM (in the range $0.3-2 \mathrm{keV}$ ). The INTEGRAL data indicate that the upper limit in $30-50 \mathrm{keV}$ range is a factor $\approx 1.5$ above the mean RXTE spectrum and the

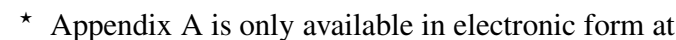
http://www. aanda.org non-thermal mechanisms are expected to contribute $\sim 50 \%$ of the total flux in this region.

The hard X-ray excess has been interpreted as being due either to inverse Compton (IC) scattering of relic CMB photons by relativistic electrons (see, e.g., Sarazin \& Lieu 1998; Fusco-Femiano et al. 1999; Brunetti et al. 2001) or to bremsstrahlung of nonthermal subrelativistic electrons (Enßlin et al. 1999; Kempner \& Sarazin 2000). Yet further interpretations have been proposed - X-ray emission by secondary electrons (Blasi \& Colafrancesco 1999), or bremsstrahlung emission by subrelativistic protons (Dogiel 2001).

Each of these models has serious problems. In the framework of the IC model the magnetic field strength can be estimated from the ratio of $\mathrm{X}$-ray to radio fluxes because both radiations are produced by the same relativistic electrons. A weak (uniform) value of the magnetic field of order $0.1-0.2 \mu \mathrm{G}$ is derived from the IC model. On the other hand, estimates of the magnetic field strength in the intracluster medium determined from Faraday rotation yield much higher value of the order of $\approx 5-10 \mu \mathrm{G}$ in the cluster center (see, e.g., Clarke et al. 2001; Govoni \& Feretti 2004). However, modifications of the IC model 
such as the complex electron spectrum model (Schlickeiser et al. 1987) or the model with anisotropic pitch angle distribution of emitting particles (Petrosian 2001) may modify the estimates of the IC model quoted above.

Models where the X-rays are generated by nonthermal electron and proton bremsstrahlung are associated with an unacceptably large energy output of emitting particles (Dogiel 2001; Petrosian 2001).

However, the bremsstrahlung model has not been completely explored because emission can also be produced by the quasithermal electron component which arises naturally when particles are accelerated from the background thermal plasma. In this case a part of the spectrum is formed under the influence of both Coulomb collisions and a run-away flux of accelerated particles (Gurevich 1960). This class of models was developed by Dogiel (2000) and Liang et al. (2002), who assumed that the hard $\mathrm{X}$-ray flux from a galaxy cluster is generated in regions of electron in-situ acceleration from the thermal pool. In such models the electron distribution function develops an extended transition populated by quasi-thermal electrons. In this region, which lies between the thermal and nonthermal parts of the spectrum, the distribution function differs strongly from the Maxwellian form because it is not an equilibrium distribution (and in this sense it is not thermal), but is formed entirely by the Coulomb collisions (and therefore we cannot define it as nonthermal).

The bremsstrahlung output from the emitting particles is proportional to the lifetime of their distribution function. For electrons with totally nonthermal energies, this lifetime is about the timescale on which a single non-thermal electron suffers ionization losses. In this regime Petrosian's (2001) estimates of the energy output required to reproduce the hard X-ray excess of Coma is completely correct. However, for a given energy in the regime where the spectrum is formed by Coulomb collisions, the lifetimes of the distribution function and that of a single electron may differ dramatically. In fact, the lifetime of, e.g., an equilibrium spectrum is much larger than the characteristic lifetime of a single particle of this spectrum. Therefore, if the hard X-ray Coma flux is emitted by electrons from the quasi-thermal transfer region, a specific and more detailed analysis is necessary for such a situation.

As it was shown by Dogiel (2000) and Liang et al. (2002), if the plasma temperature is of order of several $\mathrm{keV}$, then hard $\mathrm{X}$-rays in the energy range $20-80 \mathrm{keV}$ are produced by this quasi-thermal component of the electron flux. For a fixed radiated luminosity, such as that of the hard X-ray flux from Coma, the rate of energy input into the electrons can be lower than that required in nonthermal bremsstrahlung models. We shall discuss below this energetic problem in more detail. We will show, in addition, that this acceleration process can be tested by looking at the spectral changes in the associated Sunyaev-Zeldovich (SZ) effect from the intracluster medium.

The problem here described will be analyzed in the present paper under the following assumptions:

1. the hard X-ray flux is produced by bremsstrahlung of subrelativistic electrons;

2. electrons are in-situ accelerated from background plasma by a stochastic, Fermi-II type acceleration mechanism;

3. the characteristic time of acceleration is much larger than the characteristic time of Coulomb collisions of thermal particles, i.e. a small part of background particles is accelerated. Therefore, the parameters describing the background plasma change very slowly;
4. this allows to neglect the nonlinear terms in kinetic equations;

5. the particle acceleration is investigated in subrelativistic energy range. We do not consider, therefore, acceleration of fully relativistic electrons.

The first part of the paper is focused on the energy problem. We will show that the excess of hard X-ray emission above the thermal spectrum of Coma can be produced by electron bremsstrahlung and that the necessary energy output of electrons can be smaller than in the nonthermal bremsstrahlung model. Our analysis is based on simplified analytical solutions of the kinetic equations. Nonetheless, beyond the simplified description of the problem, it shows that the crucial energy problem set by the HXR excess can be definitely alleviated.

In the second part of the paper we derive the characteristics of in-situ acceleration from the observed spectrum of X-ray emission and we calculate the distortions of the equilibrium Maxwellian spectrum due to this acceleration. We finally explore whether a signal of acceleration in the Coma halo can be indicated by the associated Sunyaev-Zeldovich effect. In order to compare our results directly with those of Petrosian (2001), we use here the same cosmological model with $H_{0}=$ $60 \mathrm{~km} \mathrm{~s}^{-1} \mathrm{Mpc}^{-1}$.

\section{Kinetic equation for electrons}

Shock acceleration is usually considered as a candidate for particle production in the intracluster medium (see, e.g., Kuo et al. 2003), and for some clusters, the available observations suggest that there are shocks (see, e.g., Markevitch et al. 2005; Fabian et al. 2006) and electrons which might be accelerated by shocks (see, e.g. Brunetti et al. 2001; Miniati et al. 2001, 2003). However, extended radio and (perhaps) X-ray emission cannot be associated with strong shocks since accelerated electrons are unable to travel large distances from their sources without loosing much of their energy.

Plasma turbulence is hence considered as a viable model for particle (re)acceleration in cluster halos. Numerical calculations show that strong turbulence can be excited in halos (see, e.g., Ricker \& Sarazin 2001). If this turbulence generates plasma waves, then a slow stochastic process can accelerate particles through their resonant interactions with the waves. This process can be described as momentum diffusion. We note that particles can also be accelerated directly by hydrodynamic turbulent or quasi-periodic flows in a manner similar to stochastic acceleration by plasma waves (see, e.g., Webb et al. 2003).

The evolution of the distribution function of particles which are scattered by electromagnetic fluctuations is described by the Fokker-Planck equation which can be transformed to the diffusion type equation by integration over particle pitch-angles, if scattering is very effective and the distribution function is quasiisotropic. For the mechanism of the in-situ acceleration from background plasma, the equation can be written in the form

$\frac{\partial f}{\partial t}+\frac{1}{p^{2}} \frac{\partial}{\partial p} p^{2}\left[\left(\frac{\mathrm{d} p}{\mathrm{~d} t}\right)_{\mathrm{C}} f-\left\{D_{\mathrm{c}}(p)+D(p)\right\} \frac{\partial f}{\partial p}\right]=0$,

where $(\mathrm{d} p / \mathrm{d} t)_{\mathrm{C}}$ and $D_{\mathrm{c}}(p)$ describe particle convection and diffusion in the momentum space due to Coulomb collisions, and $D(p)$ is the diffusion coefficient due to the stochastic acceleration. Equation (1) can be written, in a dimensionless form, as

$\frac{\partial f}{\partial \tilde{t}}-\frac{1}{\tilde{p}^{2}} \frac{\partial}{\partial \tilde{p}}\left(A(\tilde{p}) \frac{\partial f}{\partial \tilde{p}}+B(\tilde{p}) f\right)=0$, 
where $\tilde{p}=p / \sqrt{m k T}$ is the dimensionless momentum, $\tilde{t}=t v$ is the dimensionless time and $\tilde{D}_{p}(\tilde{p})=D_{p}(p) /(v m k T)$ is the diffusion coefficient. The frequency $v$ is

$v=\frac{2 \pi n c^{2} r_{\mathrm{e}}^{2} m}{\sqrt{m k T}}$

where $r_{\mathrm{e}}=e^{2} /\left(m c^{2}\right)$ is the classical electron radius. Here

$B(\tilde{p})=\tilde{p}^{2}\left(\frac{\mathrm{d} \tilde{p}}{\mathrm{~d} \tilde{t}}\right)_{i}$,

and

$A(\tilde{p})=B(\tilde{p}) \frac{\gamma}{\sqrt{\gamma^{2}-1}} \sqrt{\frac{k T}{m c^{2}}}+\tilde{p}^{2} \tilde{D}_{p}(\tilde{p})$.

The dimensionless rate of ionization loss has the form

$$
\begin{aligned}
\left(\frac{\mathrm{d} \tilde{p}}{\mathrm{~d} \tilde{t}}\right)_{i}= & \frac{1}{\tilde{p}} \sqrt{\tilde{p}^{2}+\frac{m c^{2}}{k T} \frac{\gamma}{\sqrt{\gamma^{2}-1}}} \\
& \times\left\{\ln \left[\frac{E m c^{2}\left(\gamma^{2}-1\right)}{h^{2} \omega_{\mathrm{p}}^{2} \gamma^{2}}\right]+0.43\right\},
\end{aligned}
$$

where $\omega_{\mathrm{p}}$ is plasma frequency and $E(\tilde{p})$ is the particle total energy. The quasi-steady state solution of Eq. (2), obtained by Gurevich (1960), reads

$f=\sqrt{\frac{2}{\pi}} n(\tilde{t}) \exp \left(-\int_{0}^{\tilde{p}} \frac{B(v)}{A(v)} \mathrm{d} v\right) G(\tilde{p})$,

where

$$
G(\tilde{p})=\frac{\int_{\tilde{p}}^{\infty}[\mathrm{d} v / A(v)] \exp \left(\int_{0}^{v}[B(t) / A(t)] \mathrm{d} t\right)}{\int_{0}^{\infty}[\mathrm{d} v / A(v)] \exp \left(\int_{0}^{v}[B(t) / A(t)] \mathrm{d} t\right)},
$$

and $n(t)$ describes slow variations of the background plasma density, consistently with the assumption of slow acceleration.

Detailed information on the conditions necessary to derive a reliable momentum diffusion coefficient $D(p)$ are not well determined yet. First of all, its value is determined by a spectrum of electromagnetic fluctuations $W(k)$ (where $k$ is the wavenumber of fluctuations) which is basically unknown, though new theoretical treatments of plasma turbulence (Verma 1999) or X-ray observations of clusters (see Schuecker et al. 2004) indicate a Kolmogorov-Oboukhoh type spectrum of turbulence in the large scale range, between $20 \mathrm{kpc}$ and $2.8 \mathrm{Mpc}$. Secondly, we do not know in details the ratio between the energy density of the intracluster plasma, $W_{\text {th }} \simeq 1 \mathrm{eV} \mathrm{cm}^{-3}$, and that of the magnetic field, $U_{H}$. This ratio is, moreover, totally unknown in the regions of particle acceleration. For the magnetic field strength observed in clusters (whose estimates ranges from $\sim 0.1$ to $\sim 10 \mu \mathrm{G}$, (see e.g. Carilli \& Taylor 2002, for a review), the ratio $\beta=W_{\text {th }} / U_{H}$ is in the range $\sim 0.4$ (low $\beta$ plasma) up to $\sim 1000$ (high $\beta$ plasma). Additional theoretical uncertainties come from the lack of knowledge of the mechanism through which turbulence is formed in regions of particle acceleration, whether it is developed by cascade processes (as it is in the new model of turbulence developed by Goldreich \& Sridhar 1997), by intermediate turbulence model (see, also Cho \& Lazarian 2004) or if it is due to the flux instability when a flux of particles escaping from acceleration regions excites there MHD fluctuations due to resonant interaction, as it may occur in the Galactic halo (see Dogiel et al. 1994) or near shock fronts (Ptuskin \& Zirakashvili 2005). Given all these uncertainties, we are unable to choose reliable parameters of the kinetic equations, and we have to resort to a quite general description whose overall features can be, nonetheless, tested against the available data.

In the simplest case of charge particle scattering, the momentum diffusion coefficient has the form

$D_{p}(p)=D_{0} p$

for nonrelativistic particles (see Toptygin 1985).

For the case of low- $\beta$ plasma the momentum diffusion coefficient was derived by Miller \& Steinacker (1992); Steinacker \& Miller (1992); Miller et al. (1996); Schlickeiser \& Miller (1998) for resonant particle-wave acceleration in solar corona and in the interstellar medium of the Galaxy. Its analytical form taken from Schlickeiser \& Miller (1998) is

$$
\begin{aligned}
D_{p}(p)= & \frac{\pi(j-1) c_{1}(s)}{4}\left(\frac{W_{\mathrm{t}}}{U_{H}}\right) \\
& \times \Omega\left(r_{\mathrm{L}} k_{\min }\right)^{j-1} \frac{v_{A} p^{2}}{v^{2}} \ln \frac{v}{v_{\mathrm{A}}},
\end{aligned}
$$

where $r_{\mathrm{L}}$ and $\Omega$ are the Larmor radius and cyclotron frequency of protons, $k_{\min }$ is the minimum wave number of the MHD spectrum, $W_{\mathrm{t}}$ is the total energy of the magnetic fluctuations and $U_{H}$ is the energy density of the large scale magnetic field. Here the spectrum of magnetic fluctuations is supposed to be described by a power-law, $W(k) \propto k^{-j}$, where $c_{1}(j)$ is a constant depending on the spectral index $j$.

The momentum diffusion coefficient in a high- $\beta$ plasma is determined by the spectrum of magnetic turbulence excited by stochastic plasma motion. In the approximation of strong turbulence, corresponding to high- $\beta$ plasma, its value depends on whether the accelerated particles are magnetized or unmagnetized in a random magnetic field. The coefficients of kinetic equations are determined by pair-correlations of random velocity and random magnetic fluctuations. Note that there is no resonant interaction in this case. Below we present general equations for the momentum diffusion coefficient derived by Dogiel et al. (1987). For magnetized particles the coefficient of momentum diffusion is given by

$D_{p}=6 \int_{-\infty}^{t}\left\langle V V^{\prime}\right\rangle\left\langle h h^{\prime}\right\rangle\left\langle\nabla \nabla^{\prime} h h^{\prime}\right\rangle \mathrm{d} t^{\prime}$

where $V$ is the turbulent velocity of the plasma, $\boldsymbol{h}=\boldsymbol{H} /|\boldsymbol{H}|$ is the random direction of the magnetic field line. The values of $\left\langle V V^{\prime}\right\rangle$, $\left\langle h h^{\prime}\right\rangle$, and $\left\langle\nabla \nabla^{\prime} h h^{\prime}\right\rangle$ are the pair-correlations of turbulent velocity, direction of magnetic field and its derivative, respectively. For unmagnetized particles one obtains

$D_{p}=\frac{2 e^{2}}{3 c^{2}} \int_{-\infty}^{\infty}\left\langle V^{2}\right\rangle_{\tau, v \tau}\left\langle H^{2}\right\rangle_{\tau, v \tau} \mathrm{d} \tau$

where

$\left\langle V^{2}\right\rangle_{\tau, v \tau}=\int_{-\infty}^{\infty}|V(k, \omega)|^{2} \exp (\mathrm{i}(\omega-\boldsymbol{k} \boldsymbol{v}) \tau) \mathrm{d} \omega \mathrm{d}^{3} k$.

From an estimate of the acceleration time scale, we can derive the energy density of the resonant magnetic fluctuations in the 
case of low- $\beta$ plasma and the correlation length of turbulence in the case of high- $\beta$ plasma.

At the present stage of our knowledge, we cannot prove or disprove the validity of any form of the diffusion coefficient; however, we notice that Clarke et al. (2001) found from ROSAT and radio observations that the total magnetic energy content in clusters is comparable to the total thermal energy content in the same cluster volume.

To circumvent this problem, we derive here the general characteristics of the acceleration mechanism - i.e., the characteristic time of acceleration (the dimensionless parameter $\alpha$, see below) necessary to produce the X-ray excess above the thermal distribution - from the observed flux of hard X-rays from Coma, a procedure which is independent of the details of the acceleration process.

In the following, the spectrum of turbulence is assumed to be a power-law, $W(k) \propto k^{-j}$, with $j \simeq 2$, i.e. a spectrum that is close to that derived by Schuecker et al. (2004). For this fluctuation spectrum the momentum diffusion coefficient $\tilde{D}(\tilde{p})$ is also a power-law function of $\tilde{p}$ in a confined range of momenta as follows from Miller \& Steinacker (1992); Steinacker \& Miller (1992, see Fig. 5).

The energy of accelerated particles

$W_{\mathrm{e}} \propto \int_{0}^{\infty} p^{4} f(p) \mathrm{d} p$

diverges for any reasonable power-law form of the diffusion coefficient as follows from Eq. (7). In order to avoid this divergence we should make the natural assumption that there is a cut-off in the fluctuation spectrum for a wave number $\bar{k}$ that gives the maximum momentum $\bar{p}$ of accelerated particles. We discuss the expected solution using a simple form of the momentum diffusion coefficient

$$
\bar{D}(p)= \begin{cases}\tilde{D}(\tilde{p}) & \tilde{p}<\bar{p} \\ 0 & \tilde{p}>\bar{p} .\end{cases}
$$

The boundary conditions at $\tilde{p}=\bar{p}$ are discussed in the Appendix. We set the cut-off momentum $\bar{p}$ at an energy $\bar{E}=\bar{p}^{2} / 2 m>$ $80 \mathrm{keV}$, above the range of observations, so that it does not affect the calculated bremsstrahlung spectrum ${ }^{1}$. In our calculations we take the minimum acceptable value of the cut-off energy to be $\bar{E}_{k}=110 \mathrm{keV}$. In the case of resonant interaction this energy corresponds to the minimum wave-number $k_{\min } \sim 4.3 \times 10^{-9} \mathrm{~cm}^{-1}$ in the spectrum of magnetic fluctuations $W(k)$. Therefore, the energy density of resonant waves is

$W_{t}=\int_{k_{\min }}^{\infty} W(k) \mathrm{d} k$.

In the nonrelativistic energy range, Eq. (2) is simplified significantly and takes the form

$\frac{\partial f}{\partial \tilde{t}}-\frac{1}{\tilde{p}^{2}} \frac{\partial}{\partial \tilde{p}}\left[\left(\frac{1}{\tilde{p}}+\tilde{D}(\tilde{p}) \tilde{p}^{2}\right) \frac{\partial f}{\partial \tilde{p}}+f\right]=0$.

For the qualitative analysis of the problem we consider the dimensionless diffusion coefficient in the power-law form

1 As follows from observations of the X-ray flux from the Galactic disk (see Lebrun et al. 2004), similar processes may accelerate particles up to $\lesssim 100 \mathrm{keV}$ in the interstellar medium, although one cannot exclude the possibility that electrons are accelerated to far higher energies.
$\tilde{D}(\tilde{p})=D(p) /\left(v_{1} m k T\right)=\alpha \tilde{p}^{q}$ that enable us to get simple analytical solutions of Eq. (17). Thus, for $q=1$ the solution of Eq. (17) has a simple form (Gurevich 1960)

$$
f(\tilde{p}) \propto \exp \left(\frac{\pi-2 \arctan \left(\sqrt{\alpha} \tilde{p}^{2}\right)}{4 \sqrt{\alpha}}\right)-1 .
$$

Note that here

$$
v_{1}=\frac{4 \pi n e^{4}}{(k T)^{3 / 2} \sqrt{m}} \ln \Lambda,
$$

where $\ln \Lambda$ is the Coulomb logarithm.

The dimensionless parameter $\alpha$ for Coma can be derived from observational data. Thus, Dogiel (2000) analyzed electron acceleration in the central part of Coma with the density $n \simeq 10^{-3} \mathrm{~cm}^{-3}$ while Liang et al. (2002) derived parameters of acceleration for the average gas density in the Coma halo, $n \simeq 10^{-4} \mathrm{~cm}^{-3}$. It is rather difficult to compare results of these investigations because different forms of momentum diffusion were used in these publications.

We notice that the solution (18) provides an illustrative oversimplification of the solution of Eqs. (7) which is useful in order to get rough quantitative estimates. We present in the following numerical calculations of Eq. (17) which show the time variation of the distribution function $f$ under the influence of stochastic acceleration and Coulomb collisions.

\section{Time variations of the spectrum of accelerated particles}

We solve Eq. (17) numerically in order to understand the time evolution of the distribution function $f$ for a diffusion coefficient $\tilde{D}(\tilde{p})=\alpha \tilde{p}^{q}$ with $\alpha \approx 0.001$ and $q=1$. As discussed earlier, $\tilde{D}(\tilde{p})$ must have a cutoff for large enough momentum. For simplicity, we choose the cutoff momentum to be larger than the maximum momentum we used in our numerical calculations. In fact, the position of the cutoff momentum does not significantly affect the evolution of the distribution function at small momenta.

We considered two initial cases: i) transformation of the Maxwellian distribution under the influence of stochastic acceleration. In this case we can estimate the characteristic time for the formation of the particle excess above the thermal distribution; ii) transformation of the resulting nonequilibrium spectrum under the influence of Coulomb collisions only.

These calculations allow us to estimate the power of the electron source necessary to compensate collisional dissipation and to keep the particle excess above the thermal distribution at the level necessary for the production of the observed hard X-ray emission in Coma.

In both cases, we calculate the evolution of the electron spectrum up to the dimensionless time $\tilde{t}=4000$ (which is equal to 1.85 Gyr for Coma, where $v_{1} \approx 7.2 \times 10^{-14} \mathrm{~s}^{-1}$ ). As for the boundary conditions, we use a free boundary condition at the high-momentum boundary of our calculations, while at the low momentum boundary we used zero-flux boundary conditions.

Figure 1 shows the temporal evolution of the distribution function $f$ formed by the combined effects of stochastic acceleration and thermalization due to the Coulomb collisions. The distribution function is normalized to the value at the low momentum boundary. The dashed curve shown in this figure is the original Maxwellian distribution, and the dotted curve is the steady state solution (with a cutoff at large momentum). The five 


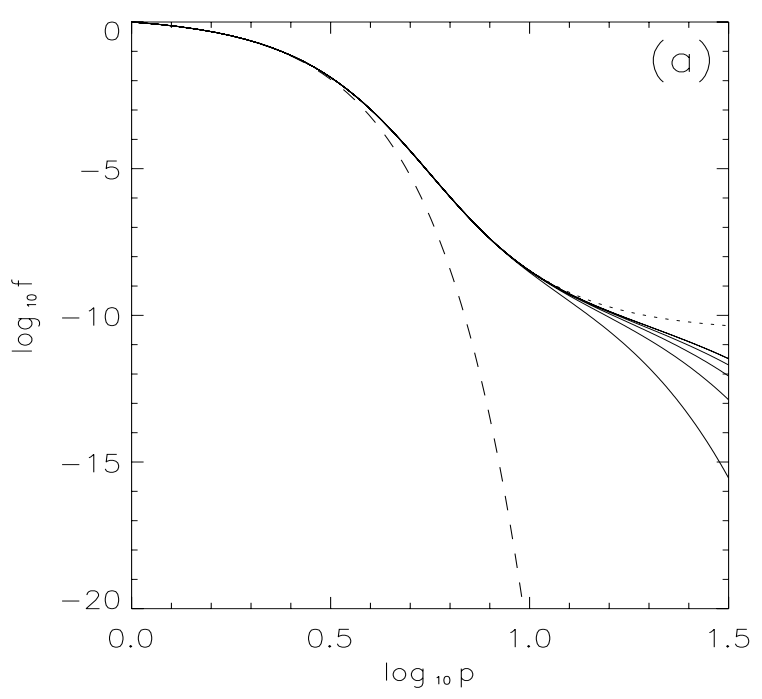

Fig. 1. Temporal evolution of the distribution function up to $\tilde{t}=4000$ $(1.85 \mathrm{Gyr})$ after acceleration is switched on. The initial distribution is Maxwellian.

solid curves represent the distribution function at increasing times $\tilde{t}=800,1600,2400,3200$, and 4000. The figure shows that the distribution function rapidly approaches the steady state solution for the relatively low momenta of the quasi-thermal particle regime ( $\tilde{p} \lesssim 10$ ). However, for nonthermal relativistic particles the stationary state is reached on timescales longer than a cluster lifetime. Therefore, in the framework of this model it is difficult to expect that subrelativistic electrons emitting hard $\mathrm{X}$-rays and relativistic electrons emitting radio emission are produced by a single mechanism of particle acceleration. Therefore, this analysis cannot be extended to relativistic energies. Our assumption that the maximum energy of the accelerated electrons is $110 \mathrm{keV}$ is hence justifiable on the basis of the previous results. Moreover, as shown by Wolfe \& Melia (2006) based on a covariant kinetic theory of electron plasmas, a power-law tail obtainable from direct or stochastic acceleration of relativistic particles cannot survive for times longer than 20 Myr because the equilibration time scale for relativistic electrons is quite short for the case of clusters and hence a thermal distribution is soon established.

Now let us assume that the acceleration mechanism is interrupted but the initial distribution function is described by the nonequilibrium form of Eq. (18). In this case, the dimensionless equation for the distribution function $f$ is

$\frac{\partial f}{\partial \tilde{t}}=\frac{1}{\tilde{p}^{2}} \frac{\partial}{\partial \tilde{p}}\left[\frac{1}{\tilde{p}} \frac{\partial f}{\partial \tilde{p}}+f\right]$.

Figure 2 shows the evolution of the electron distribution function, as described by Eq. (20), if acceleration is switched off and the distribution function is allowed to evolve from its quasisteady state form under the influence of collisional dissipation. The collisional regime of quasi-thermal particles lies in the range $5.5<\tilde{p}<30$ for $\alpha=0.001$ and $q=1$.

We show in Fig. 2 that the dissipation time scale is larger than the single electron ionization loss time scale near the thermal particle region, because the distribution function there is of almost equilibrium form, and it increases in the region of nonthermal momenta, where the lifetime of particles increases as $\tau_{i} \propto p^{3}$.

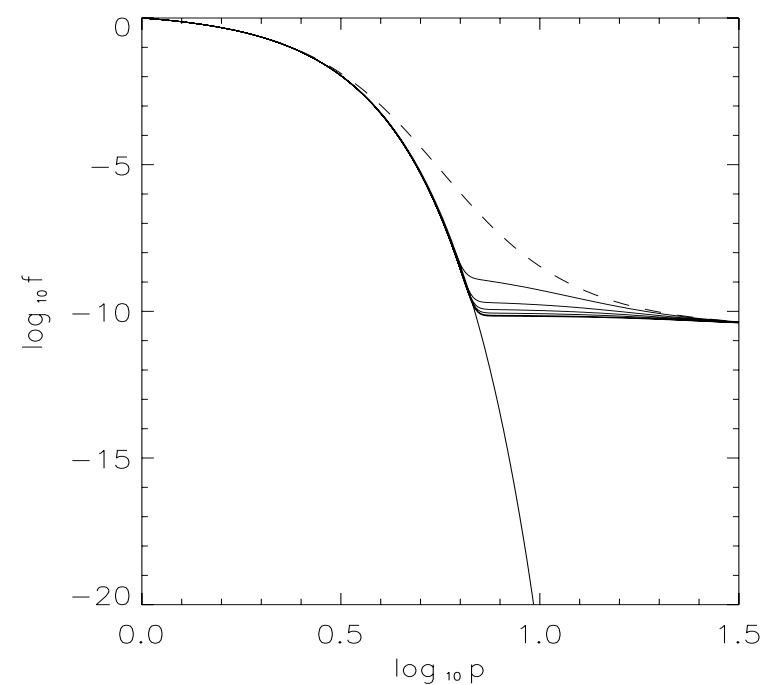

Fig. 2. Temporal evolution of the distribution function up to $\tilde{t}=4000$ (1.85 Gyr) after acceleration is switched off. The solution (18) of Eq. (2) was taken as an initial distribution function.

\section{The energetics of quasi-thermal electrons and the Coma hard X-ray flux}

We now return to the problem of the energetics of the emitting electrons. First, we recall Petrosian's criticism of the bremsstrahlung model (see Petrosian 2001). He estimated the yield of bremsstrahlung photons as $Y \sim$ $(\mathrm{d} E / \mathrm{d} t)_{\mathrm{br}} /(\mathrm{d} E / \mathrm{d} t)_{\mathrm{i}} \sim 3 \times 10^{-6}$ in the energy range $20-80 \mathrm{keV}$, where $(\mathrm{d} E / \mathrm{d} t)_{\mathrm{i}} /(\mathrm{d} E / \mathrm{d} t)_{\mathrm{br}}$ is the ratio of ionization to bremsstrahlung losses. Then for the hard X-ray flux from Coma in this energy range, $F_{\mathrm{x}} \simeq 4 \times 10^{43} \mathrm{erg} \mathrm{s}^{-1}$, a large amount of energy, $F_{\mathrm{e}} \sim F_{\mathrm{x}} / Y \sim 10^{49} \mathrm{erg} \mathrm{s}^{-1}$ is transferred from the accelerated electrons to the background plasma by ionization losses. As a result, the intracluster plasma temperature should rise to a temperature $>10^{8} \mathrm{~K}$ on a quite short time scale $\sim 3 \times$ $10^{7} \mathrm{yr}$. We stress here that these conclusions were obtained under the assumption that the lifetime of a single electron equals the lifetime of the particle distribution function. These estimates are correct only in the case that the electrons are nonthermal, and therefore collisionless. However, the previous energetic arguments cannot be used in energy ranges where the spectrum is formed by Coulomb collisions because, as we have shown in Sect. 3, the lifetime of particles differs strongly from the lifetime of the distribution function (see Fig. 3). For instance, let us consider the lifetime of thermal electrons at an energy of about $8 \mathrm{keV}$. Their individual lifetime is about $\sim 10^{6} \mathrm{yr}$, but the lifetime of the distribution function at these energies is much longer (almost infinite) because the distribution function for these energies is almost in equilibrium. Therefore, the energy supply necessary for the bremsstrahlung radiation can only be estimated from the corresponding kinetic equations. It follows that estimates of the energetics based on the lifetime of single electrons are not appropriate here, and lead to wrong conclusions. Figure 3 shows, in fact, the variation of the lifetime of the distribution function in Eq. (18), as derived from Eq. (20). From this figure we see that the lifetimes of the distribution function and of the particles are equal to each other only for high (nonthermal) electron energies.

In order to estimate the energy supply necessary to support the non-equilibrium distribution (Eq. (18)) we use the following 


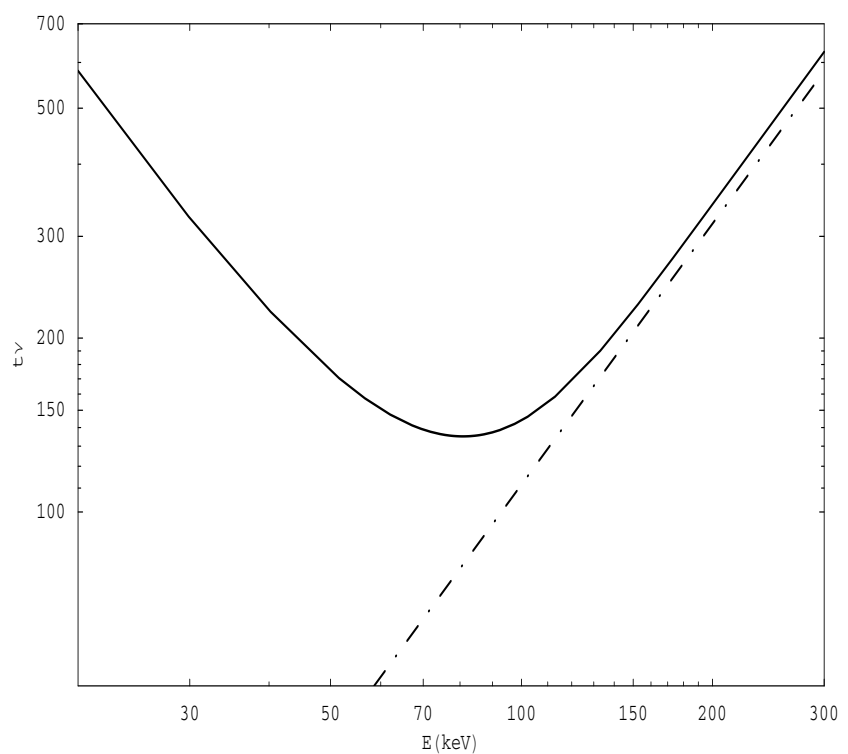

Fig. 3. The lifetime of the electron spectrum (18) derived from Eq. (17) is shown by the solid curve. The lifetime of a single electron determined by ionization loss is shown by the dashed-dotted curve. Here the dimension frequency of Coulomb collisions of thermal particles $v=7.2 \times$ $10^{-14} \mathrm{~s}^{-1}$ for the Coma parameters $T=8.2 \mathrm{keV}$ and $n=1.23 \times$ $10^{-4} \mathrm{~cm}^{-3}$.

kinetic equations in which we include bremsstrahlung losses

$$
\begin{aligned}
\frac{\partial W_{\mathrm{e}}}{\partial t} & =\Phi \\
& =4 \pi V E_{\mathrm{e}}\left(\frac{1}{p} \frac{\partial f}{\partial p}+\left(1+p^{2}\left(\frac{\mathrm{d} p}{\mathrm{~d} t}\right)_{\mathrm{br}}\right) f\right) .
\end{aligned}
$$

Here the total number of particles with momentum $\geq p$ in a volume $V$ is $F_{\mathrm{e}}(p)=V \int_{p}^{\infty} f(p) 4 \pi p^{2} \mathrm{~d} p$, and the total electron energy in this volume is $W_{\mathrm{e}} \simeq E_{\mathrm{e}} F_{\mathrm{e}}$, where the energy $E_{\mathrm{e}}=$ $k T p^{2} / 2$. Then from Eq. (20) we obtain an expression for the rate of change of the energy content of the electrons. The number of electrons emitting $50 \mathrm{keV}$ radiation is fixed in order to satisfy the observations.

In the case of nonthermal electrons whose spectrum is, e.g., a power-law, the last term exceeds the others on the right-hand side of Eq. (21), and then

$$
\left(\frac{\partial W_{\mathrm{e}}}{\partial t}\right)_{\text {nth }}=\Phi_{0} \simeq 4 \pi V E_{\mathrm{e}} f \sim \frac{W_{\mathrm{e}}}{\tau_{\mathrm{i}}},
$$

which is the result discussed by Petrosian (2001), where the electron energy loss rate is determined by the ionization loss of nonthermal particles. Here $\tau_{\mathrm{i}}$ is the characteristic time scale of the ionization loss at the energy $E_{\mathrm{e}}$.

If the particles are thermal, and their spectrum is described by a Maxwellian, then the first and the third terms on the righthand side of Eq. (20) cancel out so that

$$
\begin{aligned}
\left(\frac{\partial W_{\mathrm{e}}}{\partial t}\right)_{\mathrm{th}} \sim & 4 \pi V E_{\mathrm{e}} p^{2}\left(\frac{\mathrm{d} p}{\mathrm{~d} t}\right)_{\mathrm{br}} \frac{\partial f}{\partial p} \\
& \sim \frac{W_{\mathrm{e}}}{\tau_{\mathrm{br}}} \sim \Phi_{0} \frac{(\mathrm{d} E / \mathrm{d} t)_{\mathrm{i}}}{(\mathrm{d} E / \mathrm{d} t)_{\mathrm{br}}} .
\end{aligned}
$$

Here, the time-scale $\tau_{b r}$ is the characteristic time for bremsstrahlung loss, and $\tau_{\mathrm{br}} \gg \tau_{\mathrm{i}}$. Hence, $\left(\mathrm{d} W_{\mathrm{e}} / \mathrm{d} t\right)_{\text {nth }} \gg$ $\left(\mathrm{d} W_{\mathrm{e}} / \mathrm{d} t\right)_{\mathrm{th}}$ for the same flux of bremsstrahlung radiation produced by these electrons.

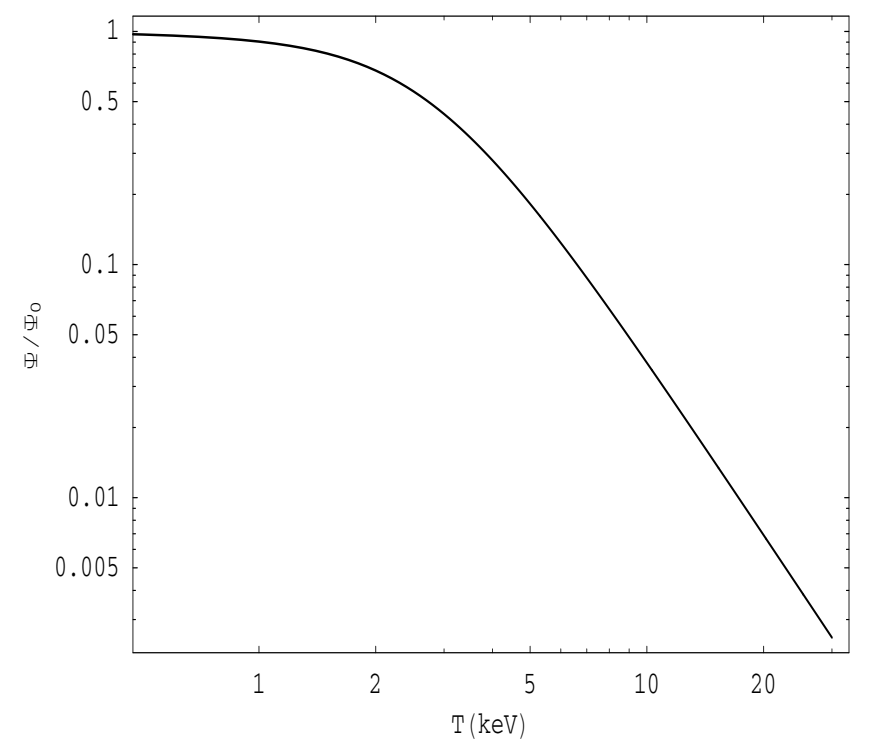

Fig. 4. The rate at which energy must be supplied to the 50-keV electrons generating a fixed bremsstrahlung X-ray flux as a function of temperature of background gas, normalized to $\Phi_{0}$, the rate at which energy must be supplied if the emitting electrons are non-thermal. For Coma $\Phi_{0} \simeq 10^{49} \mathrm{erg} \mathrm{s}^{-1}$ (Petrosian 2001).

We can estimate the rate of energy supply to the $50-\mathrm{keV}$ electrons that is required to generate the observed flux of $\sim 50-\mathrm{keV}$ bremsstrahlung X-ray emission from Coma as a function of different values of the plasma temperature $T$. For simplicity, we consider the electron spectrum to be of the form given by Eq. (18). When the temperature $T$ is low, the $50-\mathrm{keV}$ electrons are in the nonthermal particle regime, while for high plasma temperature these electrons are thermal. From Eqs. (18) and (21) we obtain the variation of the associated rate of electron heating as a function of the background temperature $T$. The required level of heating, normalized to the rate of heating required for nonthermal particles, $\Phi_{0}=\left(\mathrm{d} W_{\mathrm{e}} / \mathrm{d} t\right)_{\mathrm{nth}}$, is shown as a function of the temperature in Fig. 4, where the parameters defining the acceleration of particles are those inferred from the X-ray analysis of the Coma cluster. We find that when the temperature $T$ is low, the 50-keV electrons are nonthermal and - as expected - the rate at which they must be heated is independent of the temperature and is almost equal to $\Phi_{0}$. However, when the plasma temperature $T$ increases, and the $50-\mathrm{keV}$ electrons shift into the quasithermal regime (where the spectrum is formed by the Coulomb collisions), the required energy supply to maintain the observed hard X-ray flux from Coma decreases rapidly as the background plasma temperature increases. For a temperature $T \sim 8 \mathrm{keV}$, the emitting electrons in Coma are quasi-thermal and the energy supply they require is one or two orders of magnitude below Petrosian's (2001) result, even by using a simple qualitative estimate. Accurate quantitative calculations may give even higher variations. This result may indeed solve the energetic problem raised by Petrosian (2001): in fact, the quasi-thermal electrons need much less energy in order to produce the observed HXR bremsstrahlung radiation with respect to the case of nonthermal particles.

As it is clear from our analysis, our model describes the processe of in-situ acceleration reasonably well for relatively long time because of the presence of weak acceleration mechanisms. Therefore, the time variations of both the plasma density and of its temperature are very slow. Attempts to investigate a 


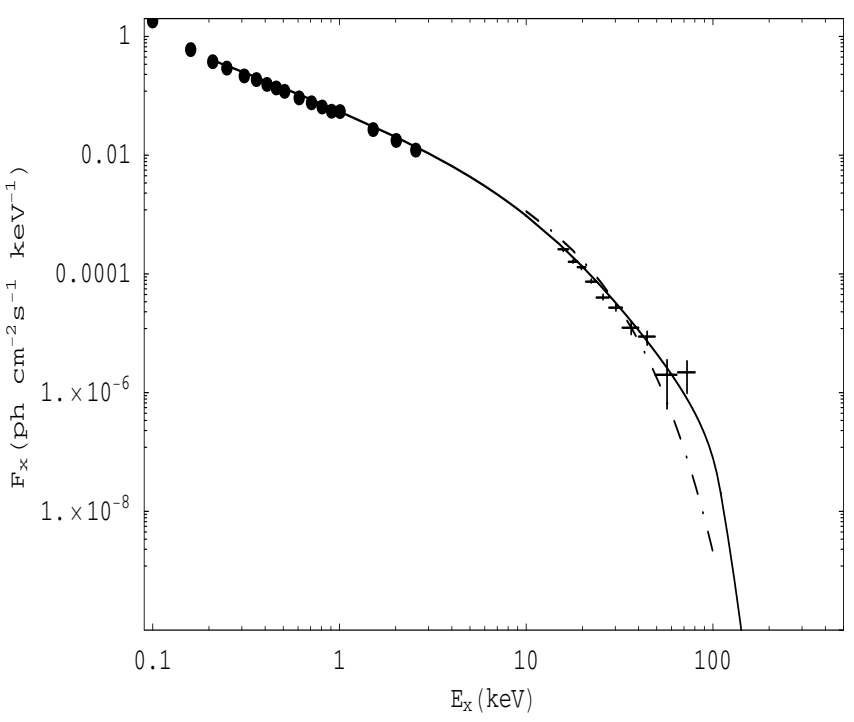

Fig. 5. Bremsstrahlung X-ray flux from the Coma cluster produced by subrelativistic electrons with a cut-off, $E=110 \mathrm{keV}, T=8.25 \mathrm{keV}$. The flux measured with Beppo-SAX was taken from Fusco-Femiano et al. (1999).

nonlinear phenomenological model of particle acceleration in Coma were made by Blasi (2000). Such an analysis showed that, due to nonlinear processes, the temperature of plasma increases slowly. However, there are still questions on whether this model can describe correctly the process of thermalization in the cluster atmospheres (see discussion by Wolfe \& Melia 2006). The role of non-linear effects certainly needs further analysis which goes beyond the scope of this paper and we will address this issue elsewhere.

\section{Bremsstrahlung emission of quasi-thermal electrons}

We derive here the spectrum of the emitting electrons in Coma from the X-ray data using the more accurate solution (see Eq. (7)) of the kinetic equation Eq. (2). We calculate the flux of hard X-ray emission using the equation

$F_{\mathrm{x}}=\frac{V}{4 \pi d_{\mathrm{L}}^{2}} \int_{E_{\mathrm{X}}}^{\infty} n v \frac{\mathrm{d} \sigma_{\mathrm{x}}}{\mathrm{d} E_{\mathrm{x}}} N(E) \mathrm{d} E$,

with cross-section

$$
\begin{aligned}
\frac{\mathrm{d} \sigma_{\mathrm{x}}}{\mathrm{d} E_{\mathrm{x}}}= & \frac{16}{3} \frac{e^{2}}{\hbar c^{2}} \frac{r_{\mathrm{e}}^{2} m c^{2}}{E E_{\mathrm{x}}} \\
& \times \ln \left(\frac{\sqrt{E}+\sqrt{E+E_{\mathrm{x}}}}{\sqrt{E_{\mathrm{x}}}}\right) .
\end{aligned}
$$

The calculated bremsstrahlung spectrum from Coma is shown together with the Beppo-SAX data in Fig. 5. We consider a the volume of particle acceleration to be $V \simeq 7.74 \times 10^{74} \mathrm{~cm}^{3}$ which is smaller than the whole volume of the Coma halo inferred from radio and soft X-ray data. In the general case it is convenient using the simplest form of the diffusion coefficient in Eq. (9). The necessary acceleration time of $\sim \mathrm{keV}$ particles, $\tau_{\mathrm{acc}}=p^{2} / D(p)$, is of the order $\sim 10^{17} \mathrm{~s}$, independently of the acceleration mechanism. This value is slightly lower than that derived by Dogiel (2000) for the central region of Coma where the intracluster gas has the highest density.

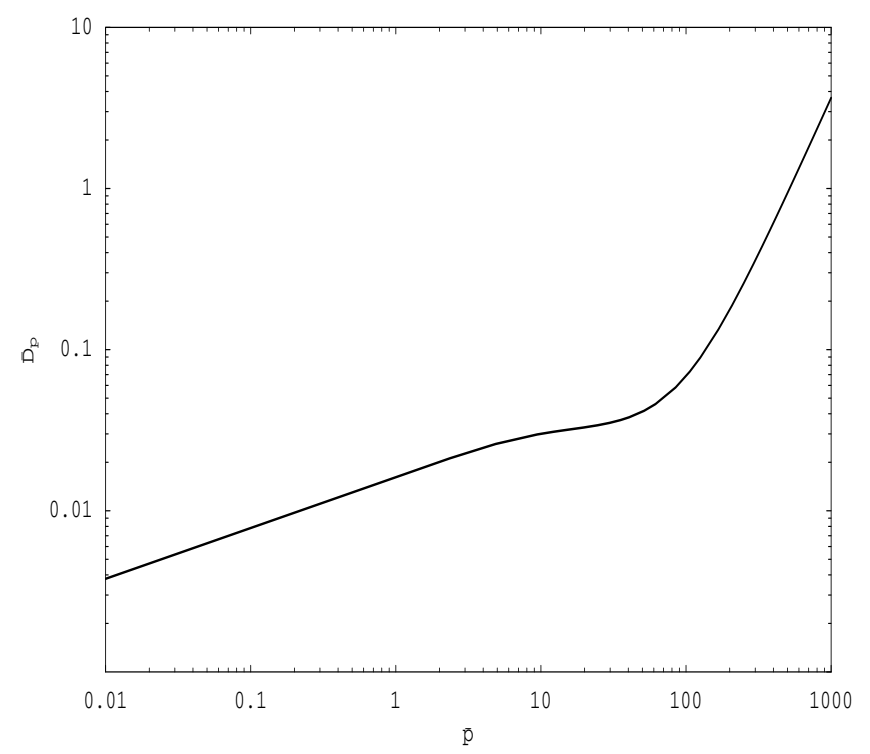

Fig. 6. The dimensionless momentum diffusion coefficient $\tilde{D}(p)=$ $D(p) /(v m k T)$ is shown as a function of the dimensionless momentum $\tilde{p}=p / \sqrt{m k T}$.

For the case of low- $\beta$ plasma we can go a step further and estimate the necessary density of resonant wave by using the diffusion coefficients for electrons and protons taken from Steinacker \& Miller (1992) and Schlickeiser \& Miller (1998). We notice that the momentum diffusion coefficient derived from these equations corresponds to the general form (9) in the nonrelativistic energy range.

Figure 6 shows the momentum diffusion coefficient for electrons for the low- $\beta$ case. Only a low energy density in resonant plasma waves, $\left(W_{\mathrm{t}} / U_{H}\right) \simeq 3.8 \times 10^{-13}$, is required to fit the Coma HXR data. Here $W_{\mathrm{t}}$ is the energy density of plasma waves and $U_{H}=H_{0}^{2} / 8 \pi$ is the energy density of the large scale magnetic field.

The corresponding electron distribution function is compared with a thermal spectrum at $T=8.25 \mathrm{keV}$ in Fig. 7. The change in the distribution function is achieved by $\sim 10 \%$ of the background electrons being quasi-thermal. It follows that the energy density of thermal electrons is $W_{\text {thermal }} \sim 1.6 \mathrm{eV} \mathrm{cm}^{-3}$ and that of the quasi-thermal electrons is $W_{\text {nonthermal }} \sim 0.56 \mathrm{eV} \mathrm{cm}^{-3}$, thus yielding the ratio $W_{\text {nonthermal }} / W_{\text {thermal }} \approx 0.35$ for Coma.

\section{The spectrum of accelerated protons}

Protons are generally disregarded in the calculation of bremsstrahlung emission because, having low rates of energy loss, they leave the emitting region before they lose a significant fraction of their energy. On the other hand, if protons escape from a radiating region relatively slowly, they can produce a bremsstrahlung flux whose value is comparable with that of the electrons. An analysis of the bremsstrahlung radiation emitted by subrelativistic protons (inverse bremsstrahlung) in the Coma cluster (Dogiel 2001) showed that protons are able, in fact, to generate the observed hard X-ray emission. These X-rays are accompanied by the excitation of background nuclei which could be detectable through the development of prominent carbon and oxygen gamma-ray lines. Dogiel (2001) estimated the expected flux of these lines. Recently, Iyudin et al. (2004) have found tracers of this gamma-ray line emission towards the Coma and Virgo clusters at the expected level. The energy deposition by 


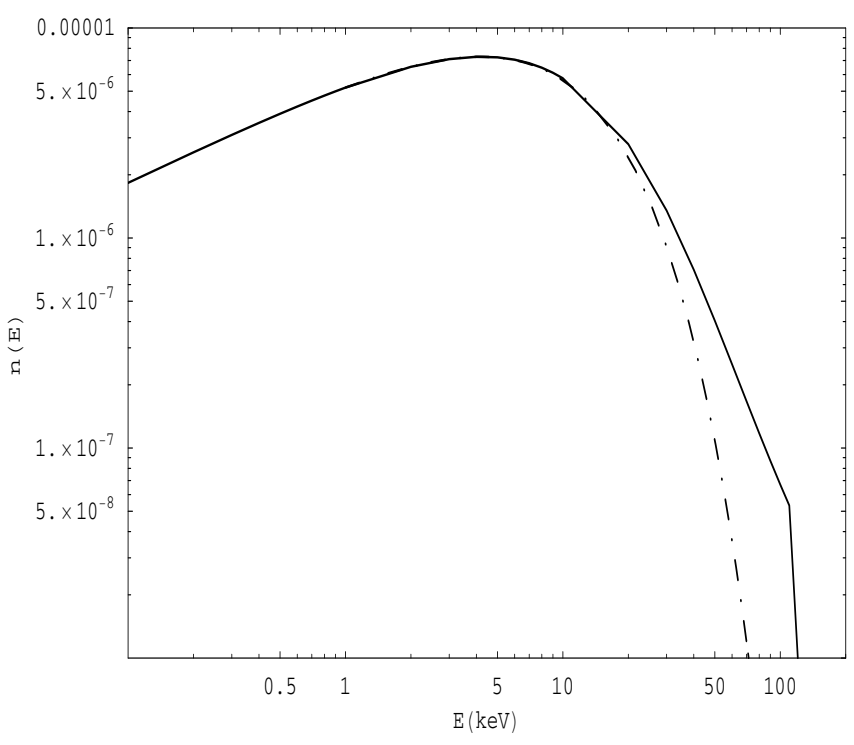

Fig. 7. Spectrum of subrelativistic electrons with cut-off, $\bar{p}=110 \mathrm{keV}$, and $T=8.25 \mathrm{keV}$ derived from the X-ray flux from the Coma cluster (solid curve). The thermal spectrum is shown by the dashed-dotted curve.

subrelativistic protons estimated by Iyudin et al. (2004) is of the order of $\sim 8 \times 10^{48} \mathrm{erg} \mathrm{s}^{-1}$, which matches the required rate if protons produce the observed hard X-ray flux (Dogiel 2001). However, this process involves subrelativistic protons and so it faces the same problem of energetics and plasma heating as we discussed in Sect. 4.

The theoretical approach we have taken in the present paper allows us to estimate the density of electrons and protons accelerated from the background pool using the kinetic parameters derived from the spectrum of plasma waves, and hence to understand whether protons are important. The momentum diffusion coefficient for protons was taken in the form of Eq. (10). In Fig. 8 the spectrum of protons is shown for the parameters derived in Sect. 5. It can be seen that stochastic acceleration is ineffective for subrelativistic protons (note that a similar conclusion for the Galaxy was also obtained by Schlickeiser \& Miller 1998). In order to produce a flux of proton bremsstrahlung comparable with that of electrons, for example at a photon energy $10 \mathrm{keV}$, the density of $20-\mathrm{MeV}$ protons should be about the same as the density of $10-\mathrm{keV}$ electrons. As shown Fig. 8, this condition is not fulfilled, and so we conclude that the hard-X-ray emission from the Coma cluster cannot be ascribed to proton bremsstrahlung.

\section{The Sunyaev-Zeldovich effect produced by in-situ accelerated particles}

In the context of the present study it is important to find observational resources which can provide independent evidence for the subrelativistic electron population that we consider to be responsible for the hard X-ray emission from clusters of galaxies. One possibility is to use a detailed analysis of the inverse Compton scattering of CMB photons off the population of subrelativistic electrons, the SZ effect described by Zeldovich \& Sunyaev (see Zeldovich \& Sunyaev 1969). The amplitude and the spectrum of this effect depend on the distribution function of the electrons in the intracluster medium. As a result of the inverse Compton scattering, the spectrum of the CMB radiation is shifted to higher frequencies when observed along the line of sight through the intracluster medium (see for a general

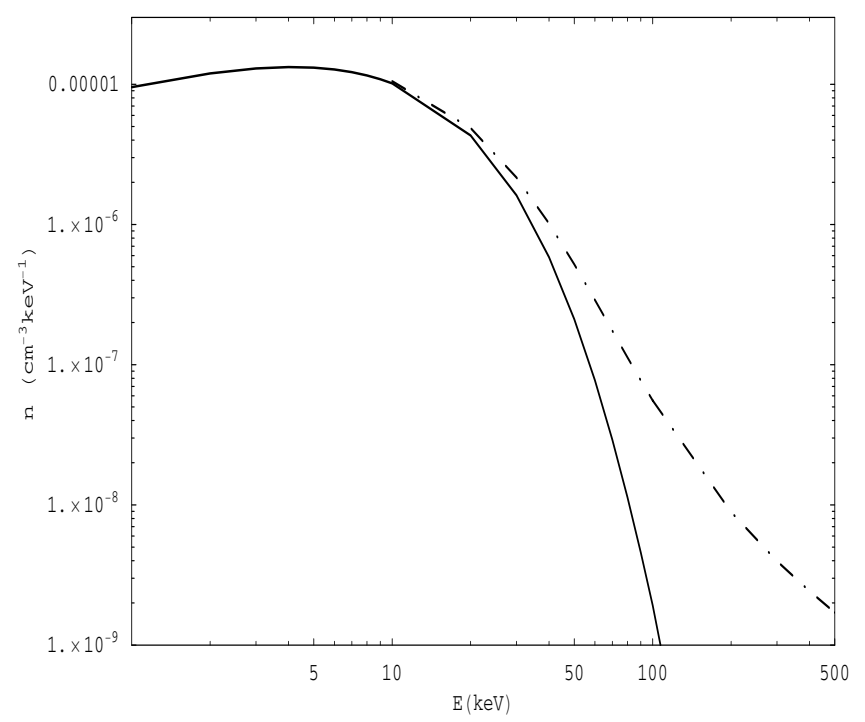

Fig. 8. The spectrum of subrelativistic electrons (dashed-dotted curve) and protons (solid curve) in the Coma cluster halo are shown as a function of the particle energy.

review Birkinshaw 1999). The spectral distortion of the microwave background can be calculated for any electron distribution function, and not only for the standard Maxwellian spectrum adopted in most discussions of the Sunyaev-Zeldovich effect: hence, we can calculate the Sunyaev-Zeldovich effect as due to a nonthermal component of the electron distribution in the suprathermal (see, e.g., Blasi et al. 2000; Ensslin \& Kaiser 2000) and relativistic (see Shimon \& Rephaeli 2002; Colafrancesco et al. 2003) energy ranges. To this aim, we use here the electron distribution function derived from the X-ray data to calculate the Sunyaev-Zeldovich effect using the formalism presented in Rephaeli (1995) and Birkinshaw (1999). The change in the radiation temperature $\Delta T(v)$ at frequency $v$ is given by

$\frac{\Delta T(v)}{T_{\mathrm{rad}}}=\frac{\left(\mathrm{e}^{x}-1\right)^{2}}{x^{4} \mathrm{e}^{x}} \frac{\Delta I(v)}{I_{0}}$,

where $x=h v / k T_{\text {rad }}$ is the dimensionless frequency, $\Delta I$ is the scattering-induced change in the specific intensity of the cosmic microwave background spectrum at frequency $v, T_{\text {rad }}=2.73 \mathrm{~K}$ is the temperature of the microwave background radiation, and the scale of the specific intensity is

$I_{0}=\frac{2\left(k T_{\mathrm{rad}}\right)^{3}}{(h c)^{2}}$.

The CMB temperature variation due to the SZ effect is

$$
\begin{aligned}
\Delta T(v)= & T_{\operatorname{rad}} \tau \frac{\left(\mathrm{e}^{x}-1\right)^{2}}{\mathrm{e}^{x} x} \int_{-\infty}^{\infty} \mathrm{d} s P_{1}(s) \\
& \times\left(\frac{\exp [-3 s)]}{\exp \left[x \mathrm{e}^{(-s)}\right]-1}-\frac{1}{\exp [x]-1}\right),
\end{aligned}
$$

where $\tau=\sigma_{\mathrm{Th}} \int \mathrm{d} \ln _{\mathrm{e}}$ is the optical depth along a line of sight of length $l$ through electrons of density $n_{\mathrm{e}}$, with $\sigma_{\mathrm{Th}}$ being is the Thomson cross-section, and

$P_{1}(s)=\int_{\beta_{\min }}^{1} p_{\mathrm{e}}(\beta) P(s, \beta) \mathrm{d} \beta$ 


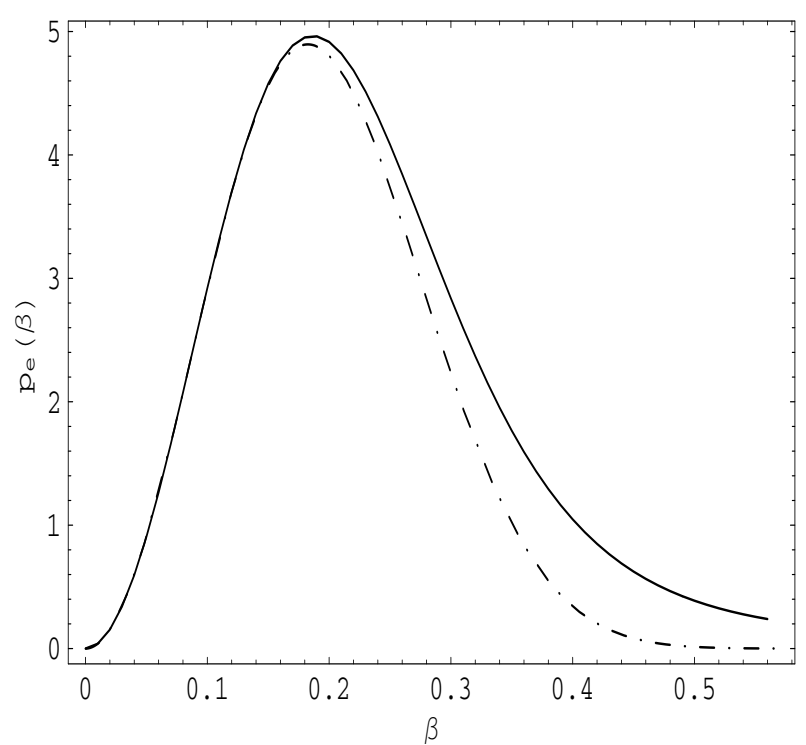

Fig. 9. The dimensionless distribution function for subrelativistic electrons with cut-off $\bar{p}=110 \mathrm{keV}$ and $T=8.25 \mathrm{keV}$ derived from the $\mathrm{X}$-ray flux from the Coma cluster (solid curve). The pure thermal spectrum is shown by the dashed-dotted curve.

is the photon redistribution function calculated in the limit of single scattering (appropriate here for low values of $\tau$ ), with

$\beta_{\min }=\frac{\mathrm{e}^{|s|}-1}{\mathrm{e}^{|s|}+1}$.

The quantity $p_{\mathrm{e}}(\beta)$ is the normalized electron spectrum given as a function of the normalized velocity $\beta=v / c$,

$\int_{0}^{1} p_{\mathrm{e}}(\beta) \mathrm{d} \beta=1$.

The function $P(s, \beta)$ describes the logarithmic frequency ratio caused by a single electron/photon scattering, and it is given in Birkinshaw (1999). The general description of the non-thermal SZ effect for multiple scattering, general values of $\tau$ and multiple electron distribution can be found in Colafrancesco et al. (2003). For practical purposes, we transform the electron distribution function from its momentum representation (Fig. 7) to its $\beta$ representation (Fig. 9). The suprathermal excess of electrons which is evident in these figures is compensated by a reduction in the number of electrons at lower energies, but this reduction is a small fractional change of the thermal electron number, and so it is neglected in Figs. 7 and 9.

We can now calculate the Sunyaev-Zeldovich effect from the regions where the acceleration takes place. Here we should take into account that, as follows from the soft X-ray data, the volume of the acceleration region, $V_{\text {acc }}=7.7 \times 10^{74} \mathrm{~cm}^{3}$, is comparable with the Beppo-SAX estimate of the emitting volume $V_{\mathrm{em}} \leq 1.7 \times 10^{75} \mathrm{~cm}^{3}$. The total optical depth of the thermal gas in the Coma cluster is not precisely known. Battistelli et al. (2003) estimated the total optical depth of Coma as $\tau_{\text {th }}=$ $(5.35 \pm 0.67) \times 10^{-3}$ and De Petris et al. (2002) estimated the depth as $\tau_{\text {th }}=(4.1 \pm 0.9) \times 10^{-3}$. Moreover, some contribution to the optical depth may be due to an extended halo of cooler gas, with a temperature $0.6-1.3 \mathrm{keV}$ and density of $10^{-4}-10^{-3} \mathrm{~cm}^{-3}$ (see Nevalainen et al. 2004).

We calculate here the Sunyaev-Zeldovich effects from the thermal volume of the Coma halo in the absence of particle acceleration (the dashed curve in Fig. 10) and in its presence (the

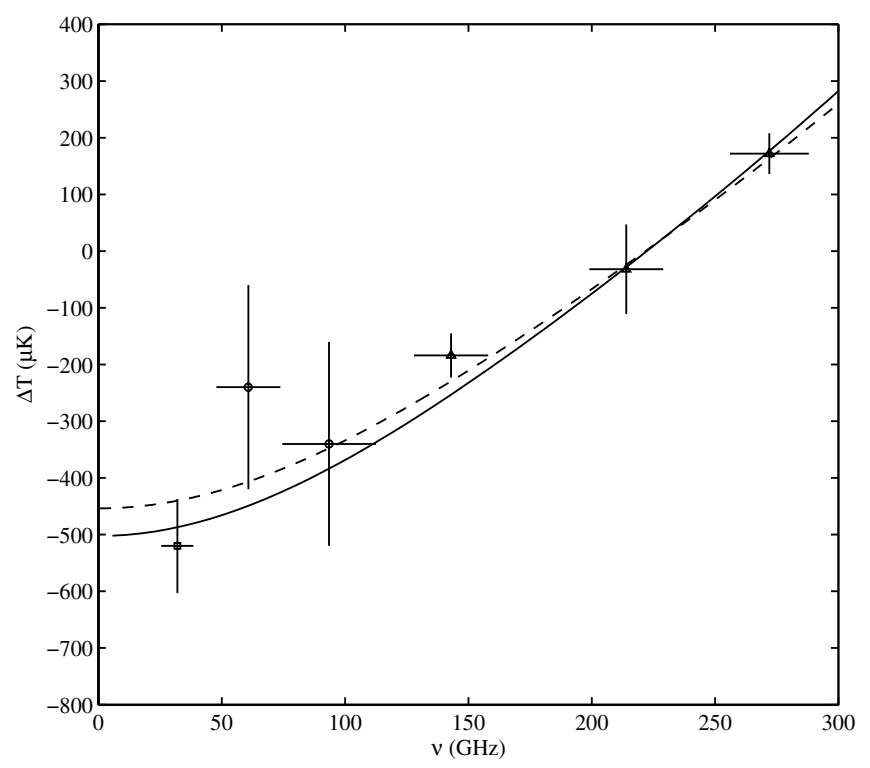

Fig. 10. The Sunyaev-Zeldovich effect produced by thermal electrons in the Coma cluster region, with temperature $T=8.25 \mathrm{keV}$ and optical depth $\tau=5.3 \times 10^{-3}$ (dashed curve). The solid curve shows the Sunyaev-Zeldovich effect due to thermal electrons from the Coma halo plus thermal and quasi-thermal electrons from regions of particle acceleration which occupy a small fraction of the Coma halo and have optical depth $9 \times 10^{-4}$ ).

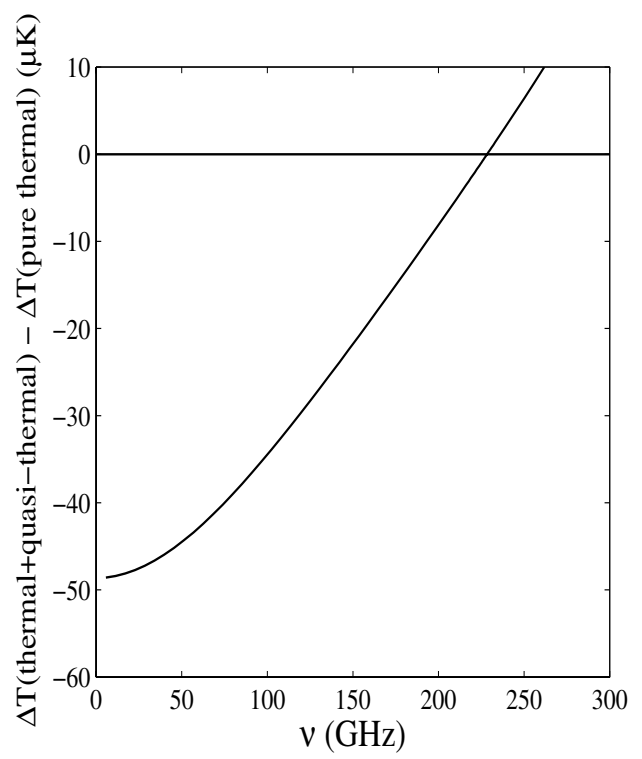

Fig. 11. Difference between the pure thermal and the total (thermal plus quasi-thermal) Sunyaev-Zeldovich effect.

solid curve in Fig. 10). In these calculations we assumed that the temperature and the optical depth of the thermal electron population are $T=8.25 \mathrm{keV}$ and $\tau_{\text {th }}=5.3 \times 10^{-3}$, as derived from X-ray observations. Such an estimate assumes that the hot plasma occupies the whole volume of the Coma halo. Assuming that the volume where particles are accelerated is $V_{\text {acc }}=7.7 \times$ $10^{74} \mathrm{~cm}^{3}$, we estimate an optical depth of the acceleration region (i.e., a part of the total optical depth where the electron spectrum is distorted by the acceleration) which is $\tau_{\text {acc }} \approx 9 \times 10^{-4}$.

The difference between the pure thermal and the total (thermal plus quasi-thermal) Sunyaev-Zeldovich effects is shown in Fig. 11. This figure shows that the presence of sub-relativistic 
electrons in Coma produces a further temperature decrement at $v \lessgtr 230 \mathrm{GHz}$ and a further temperature increase at higher frequencies. At the same time, the additional pressure (or energy density) contributed by the sub-relativistic electrons produce an increase in the frequency of the zero point (i.e., the frequency at which $\Delta T=0$ ) of the SZ effect, a value which is uniquely determined by the overall pressure $P_{\text {tot }}=P_{\text {th }}+P_{\text {subrel }}$, of the electron population (see Colafrancesco et al. 2003). Also the overall amplitude of the temperature decrement due to the subrelativistic electrons is proportional to their pressure since $\Delta T \propto \int \mathrm{d} l P_{\text {subrel }}$.

The change in the Sunyaev-Zel'dovich effect due to the accelerated electrons produces a temperature decrement $\Delta T \sim$ $50-40 \mu \mathrm{K}$ in the frequency range $50-100 \mathrm{GHz}$. However, the uncertainty of the available SZ data for Coma does not allow yet to set definite constraints on the model presented in this paper. Nonetheless, with the new generation of telescopes for observing the Sunyaev-Zel'dovich effect, such a signal should be detectable. The major difficulty in seeing this departure from the thermal Sunyaev-Zel'dovich effect is the presence of background fluctuations in the microwave background radiation. The expected background anisotropy on degree angular scales of interest for the Coma cluster is $\sim 40 \mu \mathrm{K}$ in this frequency range, and has a flat spectrum which is not readily distinguished from the gently-curving spectrum seen in Figs. 10, 11. However, the use of multi frequency observations increases the possibility of detection of the SZ effect associated to sub-relativistic particles since the relative amplitudes of the thermal and subrelativistic SZ effects change with the frequency over the whole range $(\sim 30-300 \mathrm{GHz})$ accessible to $\mathrm{SZ}$ experiments. Some possibility of detecting the distorted spectrum of the SunyaevZel'dovich effect in the presence of background fluctuations also exists through a detailed spectral measurement of the cluster over a smaller region to reduce the background fluctuation signal (although this won't reduce the amplitude of the kinematic Sunyaev-Zel'dovich effect, which has the same spectrum as the background fluctuations). Measurements of this type are challenging, but should become possible as the sensitivity of bolometer arrays in the $\mathrm{mm}$ and sub-mm bands increases, provided that accurate cross-calibration in the different bands is achieved (Birkinshaw \& Lancaster 2005).

The possibility to measure the SZ effect from the population of sub-relativistic or relativistic particles could provide a way to estimate the overall pressure of such particle population and, in turn, to constrain the energy spectrum taken up by such particles in the cluster atmosphere. Even if this demands a large experimental effort, the result will undoubtedly shed additional, and maybe crucial, light on the nature of the acceleration mechanism.

\section{Conclusions}

We have analyzed in this paper the process of particle acceleration from a background plasma acting through magnetic fluctuations generated by intracluster turbulence with a specific application to the problem of the origin of hard X-ray emission from the Coma cluster. From the equations describing the influence of Coulomb collisions, we derived the entire electron distribution function from the thermal to the high-energy nonthermal regime. Our analysis allowed us to estimate the energy supply necessary for bremsstrahlung to be responsible for the hard X-ray emission. For nonthermal electrons we confirm that the bremsstrahlung efficiency is low, which makes it almost impossible to regard this electronic component as the source of the hard X-ray excess in Coma. This result is in complete agreement with earlier conclusion (see Petrosian 2001) on the inapplicability of the nonthermal bremsstrahlung interpretation. However, we have also shown here that the bremsstrahlung efficiency increases significantly if the emitting electrons belong to the extended transfer regime between the thermal and nonthermal parts of the electron distribution function. This quasi-thermal regime is formed naturally when emitting particles are accelerated from the background plasma. In the specific case of Coma, we found that the total energy loss rate of the quasi-thermal electrons that emit the HXR radiation in the $20-80 \mathrm{keV}$ range is almost two orders of magnitude lower than for nonthermal particles. This result may solve both the problem of the origin of the HXR emission in Coma and of the excessive heating of the cluster gas in the bremsstrahlung interpretation of the HXR excess.

We have further shown that the distribution function of quasi-thermal electrons that we derived implies significant distortion of the thermal Sunyaev-Zeldovich effect from the Coma cluster. Although this additional signal is at the level of $\sim 10 \%$ of the amplitude of the thermal Sunyaev-Zel'dovich effect and therefore its observation will be challenging from the experimental side, its definite detection will nonetheless be able to provide a stringent test of our theoretical model.

Acknowledgements. The authors thank the Referee whose comments helped us to improve the paper. V.A.D. is grateful to Anisia Tang and Boris Klumov for kind help to perform numerical calculations. C.M.K. is supported in part by the National Science Council of Taiwan grants NSC-92-2112-M-008-046 and NSC-93-2112-M-008-017. P.H.K. is supported in part by the National Science Council of Taiwan grant NSC-93-2112-M-008-006. C.Y.H. is supported in part by the National Science Council of Taiwan grant NSC-93-2112-M-008-016. W.H.I. is supported in part by the National Science Council of Taiwan grants NSC-93-2112-M-008-006 and NSC-93-2752-M-008-001-PAE. V.A.D. and D.A.P. are partly supported by the grant of a President of the Russian Federation "Scientific School of Academician V. L. Ginzburg".

\section{References}

Battistelli, E. S., De Petris, M., Lamagna, L., et al. 2003, ApJ, 598, L75

Berezinskii, V. S., Bulanov, S. V., Dogiel, V. A., Ginzburg, V. L., \& Ptuskin, V. S. 1990, Astrophysics of Cosmic Rays, ed. V. L. Ginzburg, Norht-Holland; Amsterdam

Birkinshaw, M. 1999, Phys. Rep., 310, 97

Birkinshaw, M., \& Lancaster, K. 2005, Proc. 2004 Varenna Summer School Blasi, P. 2000, ApJ, 532, L9

Blasi, P., \& Colafracesco, S. 1999, Astropart. Phys., 12, 169

Blasi, P., Olinto, A. V., \& Stebbins, A. 2000, ApJ, 535, 71

Brunetti, G., Setti, G., Feretti, L., \& Giovanninni, G. 2001, MNRAS, 320, 365 Cho, Jungyeon, \& Lazarian, A. 2004, ApJ, 615, L41

Clarke, T. E., Kronberg, P. P., \& Böhringer, H. 2001, ApJ, 547, L111

Colafrancesco, S., Marchegiani, P., \& Palladino, E. 2003, A\&A, 397, 27

De Petris, M., Battistelli, E. S., D’Alba, L., et al. 2002, ApJ, 574, L119

Dogiel, V. A., Gurevich, A. V., Istomin, Ya. N., \& Zybin, K. P. 1987, MNRAS, 228,843

Dogiel, V. A. 2000, A\&A, 357, 66

Dogiel, V. A. 2001, Proc. 4th Integral Workshop, in Exploring the Gamma-Ray Universe, ed. A. Gimenez, V. Reglero, \& C. Winkler, ESA SP-459, 139

Dogiel, V. A., Gurevich, A. V., \& Zybin, K. P. 1994, A\&A, 281, 937

Enßlin, T. A., Lieu, R., \& Bierman, P. L. 1999, A\&A, 344, 409

Ensslin, T., \& Kaiser, C. 2000, A\&A, 360, 417

Fabian, A. C., Sanders, J. S., Taylor, G. B., et al. 2006, MNRAS, 366, 417 Fusco-Femiano, R., Dal Fiume, D., Feretti, L., et al. 1999, ApJ, 513, L21 Fusco-Femiano, R., Orlandi, M., Brunetti, G., et al. 2004, ApJ, 602, L73 Fusco-Femiano, R., Landi, R., \& Orlandini, M. 2005, ApJ, 624, L69 Goldreich, P., \& Sridhar, S. 1997, ApJ, 485, 680

Govoni, F., \& Feretti, L. 2004, Int. J. Mod. Phys., 13, 1549

Gurevich, A. V. 1960, Sov. Phys. JETP, 38, 1150 
Hughes, J. P., Butcher, J. A., Stewart, G. C., \& Tanaka, Y. 1993, ApJ, 404, 611 Iyudin, A. F., Böhringer, H., Dogiel, V., \& Morfill, G. 2004, A\&A, 413, 817 Kempner, J. C., \& Sarazin, C. L. 2000, ApJ, 548, 639

Kuo, Ping-Hung, Hwang, Chorng-Yuan, \& Ip, Wing-Huen 2003, ApJ, 594, 732 Kuo, Ping-Hung, Bowyer, Stuart, \& Hwang, Chorng-Yuan 2005, ApJ, 618, 675 Lebrun, F., Terrier, R., Bazzano, A., et al. 2004, Nature, 428, 293

Liang, H., Dogiel, V. A., \& Birkinshaw, M. 2002, MNRAS, 337, 567

Markevitch, M., Govoni, F., Brunetti, G., \& Jerius, D. 2005, ApJ, 627, 733

Miller, J. A., \& Steinacker, J. 1992, ApJ, 399, 284

Miller, J. A., Larosa, T. N., \& Moore, R. L. 1996, ApJ, 461, 445

Miniati, F., Ryu, Dongsu, Kang, Hyesung, \& Jones, T. W. 2001, ApJ, 559, 59

Miniati, F. 2003, MNRAS, 342, 1009

Nevalainen, J., Oosterbroek, T., Bonamente, M., \& Colafrancesco, S. 2004, ApJ, 608,166

Petrosian, V. 2001, ApJ, 557, 560

Ptuskin, V. S., \& Zirakashvili, V. N. 2005, A\&A, 429, 755

Renaud, M., Belanger, G., Paul, J., \& Terrier, R. 2006,

[arXiv:astro-ph/0606114]
Rephaeli, Y. 1995, ARA\&A, 33, 541

Rephaeli, Y., \& Gruber, D. E. 2002, ApJ, 579, 587

Ricker, P. M., \& Sarazin, C. L. 2001, ApJ, 561, 621

Rossetti, M., \& Molendi, S. 2004, A\&A, 414, L41

Sarazin, C. L., \& Lieu, R. 1998, ApJ, 494, L177

Schlickeiser, R., \& Miller, J. A. 1998, ApJ, 492, 352

Schlickeiser, R., Sievers, A., \& Thielmann, H. 1987, A\&A, 182, 21

Schuecker, P., Finoguenov, A., Miniati, F., Bohringer, H., \& Briel, U. G. 2004, A\&A, 426, 387

Shimon, M., \& Rephaeli, Y. 2002, ApJ, 536, 31

Steinacker, J., \& Miller, J. A. 1992, ApJ, 393, 764

Toptygin I. N. 1985, Cosmic Rays in Interplanetary Magnetic Fields (Amsterdam: Reidel)

Verma, M. K. 1999, Phys. Plasmas, 6, 1455

Webb, G. M., Ko, C. M., Zank, G. P., \& Jokipii, J. R. 2003, ApJ, 595, 195

Wolfe, B., \& Melia, F. 2006, ApJ, 638, 125

Zeldovich, Ya. B., \& Sunyaev, R. A. 1969, Ap\&SS, 4, 301 


\section{Online Material}




\section{Appendix A: kinetic equation}

In the nonrelativistic energy range when $E \gg k T(p \gg 1)$, the kinetic equation for $p<\bar{p}$ and $p>\bar{p}$ can be significantly simplified and written in the form respectively

$\frac{\partial f}{\partial \tilde{t}}-\frac{1}{\tilde{p}^{2}} \frac{\partial}{\partial \tilde{p}}\left[\left(\frac{1}{\tilde{p}}+\alpha \tilde{p}^{q+2}\right) \frac{\partial f}{\partial \tilde{p}}+f\right]=0$,

$\frac{\partial f}{\partial \tilde{t}}-\frac{1}{\tilde{p}^{2}} \frac{\partial}{\partial \tilde{p}}\left(\frac{1}{\tilde{p}} \frac{\partial f}{\partial \tilde{p}}+f\right)=0$,

with the boundary conditions corresponding to continuity of the function

$f(\bar{p}, t)=\bar{f}(\bar{p}, t)$,

where $\bar{f}$ is the solution of Eq. (A.1) for the unknown flux of particles, $-S_{0}$, running away into the acceleration region

$$
\begin{aligned}
\bar{f}(\tilde{p}, \tilde{t})= & \sqrt{\frac{2}{\pi}} n(\tilde{t}) \exp \left[-\int_{0}^{\tilde{p}} \frac{B(v)}{A(v)} \mathrm{d} v\right] \\
& -S_{0} \int_{0}^{\tilde{p}} \frac{\mathrm{d} v}{A(v)} \exp \left[-\int_{v}^{\tilde{p}} \frac{B(t)}{A(t)} \mathrm{d} t\right] .
\end{aligned}
$$

The flux of particles running into the acceleration region derived from Eq. (A.4) is

$S(\tilde{p})=-S_{0} \sqrt{\frac{2}{\pi}} \int_{0}^{\tilde{p}} v^{2} \exp \left(-v^{2} / 2\right) \mathrm{d} v$

and this changes from $S=0$ for $\tilde{p}=0$ to $S=-S_{0}$ for $p \approx \tilde{p}_{\mathrm{M}}=\alpha^{-1 /(q+3)}$. Above the momentum $\tilde{p}_{\mathrm{M}}$, the function $f$ cannot be described by a Maxwellian distribution because the equilibrium conditions are violated by the run-away flux $S$, but the spectrum is still formed by Coulomb collisions. For energies above $\tilde{p}_{\text {inj }}=\alpha^{-1 /(q+1)}$, the spectrum is nonthermal because Coulomb collisions are unimportant particle interactions while plasma waves dominate.

It was shown by Dogiel (2000) and Liang et al. (2002) that two excesses above the thermal Maxwellian spectrum are formed in the range $\tilde{p}>\tilde{p}_{\mathrm{M}}$. When $\tilde{p}_{\mathrm{M}}<\tilde{p}<\tilde{p}_{\text {inj, }}$, the excess is formed by Coulomb collisions (the collisional regime of quasi-thermal particles), and one can imagine the spectrum there as a distorted Maxwellian function. For $p>p_{\text {inj }}$ the spectrum is formed by particle interactions with plasma waves (the collisionless regime of nonthermal particles). For these energies the particle spectrum can be described as power-law over an extended energy range. We also impose a natural boundary condition for $\tilde{p}=\infty$,

$$
f(\infty)=0 .
$$

In order to derive the unknown constant $S_{0}$ we should match solutions of Eqs. (2) and (A.2) for $\tilde{p}=\bar{p}$.

It is convenient to reduce Eq. (A.2) to the form

$\frac{\partial^{2} z}{\partial \zeta^{2}}-\frac{\partial z}{\partial t}=-z \frac{1}{4 \sqrt{\xi}}\left(\frac{9}{16} \frac{1}{\xi^{2}}-1\right)$

by introducing the variable $\zeta$,

$\zeta=2^{1 / 4} \frac{4}{5}\left(\frac{\tilde{p}^{2}}{2}\right)^{5 / 4}$, and the function $z$,

$f=z\left(\frac{\eta^{2}}{\sqrt{\ln (1 / \eta)}}\right)^{1 / 4}$

where

$\eta=\exp \left(-\tilde{p}^{2} / 2\right)$

and

$\xi=\left(\frac{5 \zeta}{2^{9 / 4}}\right)^{4 / 5}$.

For large $\zeta$ (or equivalent $p$ or $\xi$ ) values we can neglect the righthand side in Eq. (A.7), and the equation takes the well-known one-dimensional diffusion equation form

$\frac{\partial^{2} z}{\partial \zeta^{2}}-\frac{\partial z}{\partial t} \approx 0$

with boundary condition (A.3). As an initial condition we put

$z(\zeta)=0 \quad$ at $\quad t=0$

i.e., we assume that there were no particles in the momentum range $\tilde{p}>\bar{p}$ at $\tilde{t}=0$.

The solution of Eq. (A.2) can be presented with the wellknown Green function $G\left(x, x_{0} \mid t, \tau\right)$ for the diffusion equation

$G\left(x, x_{0} \mid \tilde{t}, \tau\right)=$

$$
\frac{1}{2 \sqrt{\pi}} \frac{1}{(\tilde{t}-\tau)^{1 / 2}} \exp \left[-\frac{\left(x-x_{0}\right)^{2}}{4(\tilde{t}-\tau)}\right] .
$$

Then the function $f$ for Eq. (A.12) with the boundary conditions (A.3) has the form

$$
\begin{aligned}
f(\tilde{p}, \tilde{t})= & \bar{f}(\bar{p})\left(\frac{\bar{p} \exp \left(\bar{p}^{2}\right)}{\tilde{p} \exp \left(\tilde{p}^{2}\right)}\right)^{1 / 4} \\
& \times\left[1-\Phi\left(\frac{(\zeta-\bar{\zeta})}{2 \sqrt{t}}\right)\right],
\end{aligned}
$$

where $\Phi(x)$ is the error function. We see that for $t \rightarrow \infty$ the solution (A.15) tends to the stationary distribution. In this limit $\partial f / \partial \tilde{t} \ll 1$ and our neglect of the right-hand side of Eq. (A.7) is not valid. Therefore in the stationary distribution limit, the distribution function $f$ tends to the equilibrium Maxwellian function for $\tilde{p}>\bar{p}$ when $\tilde{t} \rightarrow \infty$,

$f(\tilde{p}, \tilde{t}=\infty)=C(\tilde{t}) \exp \left[-\frac{\tilde{p}^{2}}{2}\right]$.

The constant $C$ and the unknown flux $S_{0}$ can be defined from the boundary condition (A.3) and the normalization condition

$\int_{\bar{p}}^{\infty} f(\tilde{p}) \tilde{p}^{2} \mathrm{~d} \tilde{p} \simeq S_{0} t$.

However, as we see from Eq. (A.15), in the stationary case we have an exponential cut-off of the distribution function $f$ for $\tilde{p}=$ $\bar{p}$, which gives an approximate equation for $S_{0}$ of the form

$S_{0}=\sqrt{\frac{2}{\pi}} n(\tilde{t}) \times\left(\int_{0}^{\bar{p}} \frac{\mathrm{d} v}{A(v)} \exp \left[-\int_{0}^{v} \frac{B(t)}{A(t)} \mathrm{d} t\right]\right)^{-1}$,

where $n(t)$ is the density of background plasma, which decreases slowly with time. 